\title{
Trauma-informed knowledge, awareness, practice, competence and confidence of rural health staff: A descriptive study
}

\author{
Kaye Ervin ${ }^{* 1}$, Carol Reid ${ }^{1}$, Tegan Podubinski ${ }^{1}$, Jacquie Phillips ${ }^{2}$ \\ ${ }^{1}$ The University of Melbourne, Department of Rural Health, Shepparton, Victoria, Australia \\ ${ }^{2}$ NCN Health, Nathalia, Cobram, Numurkah, Victoria, Australia
}

Received: February 2, 2021

DOI: $10.5430 /$ jnep.v11n9p1
Accepted: April 12, 2021

URL: https://doi.org/10.5430/jnep.v11n9p1
Online Published: April 22, 2021

\begin{abstract}
Background and objective: By adopting a trauma-informed approach to care at the organisational and clinical levels, health care systems and providers can enhance the quality of care that they deliver and improve health outcomes for individuals with a trauma history. This study aimed to explore the trauma-related knowledge, attitudes awareness, practice, competence and confidence of health service staff from three small rural health services in Victoria, Australia, and examine their self-reported capacity to respond to clients with a trauma history.

Methods: Staff from each site were invited to complete a paper-based survey. The survey included demographic information and questions related to knowledge and understanding of trauma, experience of trauma-informed care and confidence engaging in, and perceived importance of, trauma-informed practices.

Results: The respondents were predominately nurses. Results showed that $16 \%$ of respondents had undertaken training in trauma-informed care and $44 \%$ disagreed that they had an understanding of trauma-informed practices. There were high levels of agreement for statements related to knowledge and understanding of trauma and low levels of agreement with statements related to experience of trauma-informed care. More than $70 \%$ of respondents reported that they had little knowledge of the principals of trauma-informed care, and little experiencing with practicing trauma-informed care.

Discussion and conclusions: Overall, the survey results showed that staff were trauma-aware, but supported the need for more education and training in trauma-informed practices and improved organisational approaches to support trauma-informed approaches. It is important for organisations to shift from being trauma aware to being trauma-informed, by building foundational awareness of these practices and reinforcement through continuing education.
\end{abstract}

Key Words: Trauma-informed care, Trauma, Health service delivery, Nursing, Family violence, Homelessness

\section{INTRODUCTION}

There is no universally accepted definition of trauma. For the purposes of this study trauma is defined as a series of events or circumstances experienced by an individual that have a lasting adverse effect on mental, physical, social, emo- tional, or spiritual wellbeing. This definition is a concise definition of that provided by the expert body SAMHSA. ${ }^{[1]}$ Research suggests that exposure to adverse, potentially traumatic events in childhood, such abuse or neglect, is common. ${ }^{[2]}$ In 2015, it was reported that childhood trauma af-

\footnotetext{
*Correspondence: Kaye Ervin; Email: keervin@unimelb.edu.au; Address: Rural Health Academic Network, the University of Melbourne, Victiria, Australia.
} 
fected an estimated five million Australian adults. ${ }^{[3]}$ More recent estimations involving childhood trauma events, cite that 8.9\% of Australian children experience physical abuse, $8.6 \%$ sexual abuse, $8.7 \%$ emotional abuse and $2.4 \%$ neglect. ${ }^{[4]}$ Apart from children, two studies suggest that $57 \%-75 \%$ of Australians will experience a potentially traumatic event at some point in their lives. ${ }^{[5,6]}$ In addition, trauma exposure is more common among specific groups, such as people who experience homelessness, young people in out-of-home care, refugees, women and children experiencing family and domestic violence, LGBTIQ people, Aboriginal and Torres Strait Islander people and certain occupation groups such as emergency service workers and members of the defence forces. ${ }^{[7]}$

Trauma-informed care is a framework for service provision that is based on knowledge and understanding of how trauma affects people's lives, ${ }^{[8]}$ as well as their health service needs and is grounded in promoting a culture of being responsive to the impact of trauma and actively resisting re-traumatisation. ${ }^{[9]}$ Available literature depicts a traumainformed care continuum from becoming trauma aware, (which is seeking information about trauma and its implications for survivors and providers), to being trauma-informed (a cultural shift at the systemic level). ${ }^{[9]}$ At the most basic level, staff who are trauma aware have an understanding of trauma and how symptoms and behavioural presentations in individuals may be responses to traumatic experiences. ${ }^{[10]}$ Melz and colleagues ${ }^{[11]}$ suggest that trauma-informed services develop and sustain trauma awareness, knowledge, and skills into their organisational cultures, practices, and policies to create a climate of empathy and respect.

Previous studies show the critical need for trauma-informed care at all levels of service provision is growing, as trauma affects such a large percentage of the population, those affected are clients in a broad range of health service settings. ${ }^{[10,11]}$ While it is important for all health service staff to have an understanding of trauma and its impact, nurses are the highest number of direct care providers, and are positioned to play an integral role in trauma-informed care within health care services. ${ }^{[12]}$ Nurses have ample opportunities to influence the experience of patients and colleagues, and nursing is a critical field in which to introduce a trauma-informed approach. ${ }^{[13]}$

The findings of the study presented here, are the result of a collaboration between a university and small rural health services in Victoria, Australia to explore the knowledge, awareness and attitudes of health service staff and the capacity to respond to clients experiencing trauma. The driver for the study was an identified concern by the health services that they needed to improve responses to vulnerable groups, particularly those impacted by homelessness and family violence. Strategic localised planning has subsequently predicted increases in vulnerability as a consequence of COVID19.

\section{METHOD}

\subsection{Setting}

The research was undertaken at three rural health services in Victoria. The health services are located in the same geographical region and were amalgamated in 2019, sharing a Chief Executive Officer and Medical Director but having separate Directors of Clinical Services and clinical and nonclinical staff. Two of the health service sites employ the same number of staff and offer similar services, such as Urgent Care, Acute care, Aged care, Allied Health, Community Health, Medical, Dental, Surgical and Radiology. Two of the health services provide care to a population of approximately 5,000 people. One health service is smaller, servicing a population of approximately 2,000 people, with limited part time Allied Health services and no Radiology, Dental or Surgical services. Community Health, Urgent Care, Acute care, Aged care and Medical services at the smaller site are similar to the other health services by population. All of the health services have a research collaboration with the University of Melbourne. Levels of employment differed at each site, with 220,200 and 75 employees, respectively and a total of 475 overall.

\subsection{Participants}

All staff, clinical and non-clinical, at all three health services were invited to participate, the only eligibility criteria was employment at one of the health services.

\subsection{Recruitment}

The principal researcher attended each site in September 2020 and invited staff in person to complete a paper-based survey and place it in a deposit box (there were four deposit boxes located at various sites at each health service). The principal researcher was available in staff dining rooms at each site and actively recruited for a six day period (at two sites) and three days at one site where only 75 staff were employed.

\subsection{Ethics}

Ethical approval to conduct the study was granted by the University of Melbourne Human Ethics Advisory Group (Ethics ID Number: 2056637.1) in September 2020 and the project complied with the Declaration of Helsinki. All potential participants received a plain language statement describing the study, the voluntary nature of the study and a list of support 
services that offer counselling. The plain language statement clearly explained that there were no negative consequences for non-participation. The health services had no way of knowing which staff participated or did not participate as no identifying information was requested. Completion and return of the survey was considered consent to participate.

\subsection{Survey tool}

The survey questions were preceded with a highlighted definition of trauma - Trauma is defined as a series of events or circumstances experienced by an individual that has a lasting adverse effect on mental, physical, social, emotional or spiritual wellbeing. ${ }^{[1]}$ Demographic information collected included the number of years employed by the organisation, participants' role and gender. All staff were asked if they had participated in Trauma-Informed Practice training and training in Strengthening Hospital Responses to Family Violence (with yes or no category responses).

Section one of the survey contained 17 statements related to knowledge and understanding of trauma and traumainformed care and participants were asked to indicate their level of agreement with the statement, with response categories of disagree, undecided and agree. Two of statements had 'undecided' replaced with 'I don't know what they are' and 'I do not have experience'to provide more detail than undecided. Most of these statements were sourced from a previously validated tool ${ }^{[14]}$ with additional statements developed from reviewing the literature for relevance to this study setting. There were a further six statements for clinical staff only. The additional relevant statements regarding self-rated competence and opinions in specific aspects of trauma-informed care were included from a previously validated tool used to assess providers' knowledge, views and practice of trauma-informed care. ${ }^{[15]}$ The survey questions are shown in Table 1, and each question designated as knowledge, awareness, attitude and capacity for clarity. The survey included an open ended section for staff to leave general comments.

The final survey was not pre-tested, due to being predominantly sourced from validated tools, but it was approved for reliability and understanding by an expert in TraumaInformed care and two local social workers. The researcher was also available on site during data collection for staff to clarify understanding of questions. The survey was also intended to gather local level knowledge for relevance to the study sites.

The final section of the survey was a capacity assessment for clinical staff, which rated confidence and perceived impor-

Published by Sciedu Press tance of various processes, such as identifying risk factors for family violence or homelessness, providing referrals to services and access to resources. Clinical staff were asked to rate the level of importance for eight statements, with response categories of unimportant, undecided and important and then asked to rate their confidence in their skills and understanding for the same statements, with response categories of not confident, undecided and confident. A copy of the survey is available from the corresponding author.

\section{Results}

\subsection{Analysis}

Numerical codes were assigned for each response and all responses entered manually from the paper based surveys into SPSS version 26. ${ }^{[16]}$ Descriptive statistics are used for demographic information and reported as frequencies. Frequencies (number and percentage) are reported for sections one and two of the survey and further comparative analysis for clinical and non-clinical staff for section one was undertaken using independent Chi-square tests for independence. Statistical significance was set at .05 .

For the capacity assessment staff were asked to rate unimportant, undecided or important, which were assigned numerical 1, 2 and 3. Similarly for confidence in skills and understanding staff were asked to rate not confident, undecided and confident, with the same numerical ratings of 1,2 and 3 . The median score for confidence is subtracted from the median score for importance to calculate the gap.

\subsection{Descriptive findings}

Overall, there was a $78 \%(\mathrm{n}=389)$ response rate. Site one had an $83 \%$ response rate (166/200), site two had a $78 \%$ response rate $(172 / 220)$ and site three had a $68 \%$ response rate $(51 / 75)$.

The number of years employed at the organisation ranged from less than one year to 36 years (mean years of employment $=9$ years). Of those who responded, 254 (65\%) were clinical staff and $134(35 \%)$ were non clinical staff. The majority $(83 \%)$ identified as female. The roles held by respondents are shown in Table 1. More than half reported their role as nursing $(n=231)$.

Respondents were asked about previous training in TraumaInformed Practice and Strengthening Hospital Responses to Family Violence. There were 61 staff members (16\%) who reported previous training in Trauma-Informed Practice and $68 \%(\mathrm{n}=262)$ staff who reported undertaking training in Strengthening Hospital Responses to Family Violence. 
Table 1. Reported roles of respondents

\begin{tabular}{lll}
\hline Role & Number & Percent (\%) \\
\hline Registered nurse & 140 & 36.0 \\
Enrolled nurse & 91 & 23.4 \\
Aged care support staff & 7 & 1.8 \\
Administration & 62 & 15.9 \\
Environmental staff & 25 & 6.4 \\
Reception staff & 12 & 3.1 \\
Maintenance staff & 12 & 3.1 \\
Dental nurse & 1 & .3 \\
Medical doctor & 1 & .3 \\
Other & 38 & 9.8 \\
Total & 389 & 100.0 \\
\hline
\end{tabular}

All respondents, clinical and non-clinical, were then asked to rate their level of agreement with 17 statements related to knowledge, attitudes and awareness of Trauma-Informed Practice. The statements and the responses are shown in Table 2. Results are rounded to nearest $0.5 \%$.

There were high levels of agreement for the awareness statements and low levels of agreement with statements related to practice of trauma-informed care.

There were no statistical differences in responses between sites. As both clinical and non-clinical staff completed section one of the survey (see Table 2), results were further analysed to detect differences between the groups. There were statistical differences between the groups for nine of the statements. The statistical differences ( $p$ ) for the statements are shown in Table 3. The effect size (phi coefficient) is also shown, using Cohen's criteria ${ }^{[17]}$ of .10 for small effect, .30 for medium effect and .50 for large effect. Although there was a significant difference between the groups, the effect size was small.

Table 2. Level of agreement with trauma statements

\begin{tabular}{|c|c|c|c|}
\hline Statement & $\begin{array}{l}\text { Disagree } \\
\mathrm{n}(\%)\end{array}$ & $\begin{array}{l}\text { Undecided } \\
\text { n (\%) }\end{array}$ & $\begin{array}{l}\text { Agree } \\
\text { n (\%) }\end{array}$ \\
\hline Exposure to trauma is common (AW) & $48(12)$ & $59(15)$ & $282(73)$ \\
\hline Trauma affects physical, emotional and mental well-being (AW) & $1(.5)$ & $10(25)$ & $378(97)$ \\
\hline $\begin{array}{l}\text { Substance use issues can be indicative of past traumatic experiences or } \\
\text { adverse childhood experiences (AW) }\end{array}$ & $4(1)$ & $34(9)$ & $351(90)$ \\
\hline $\begin{array}{l}\text { Distrusting behavior can be indicative of past traumatic experiences or } \\
\text { adverse childhood experiences (AW) }\end{array}$ & $1(.5)$ & $15(4)$ & $371(95.5)$ \\
\hline $\begin{array}{l}\text { There is a connection between mental health issues and past traumatic } \\
\text { experiences or adverse childhood experiences (AW) }\end{array}$ & $2(.5)$ & $21(5.5)$ & $366(94)$ \\
\hline Re-traumatisation can occur unintentionally (AW) & 0 & $37(9.5)$ & 352 (90.5) \\
\hline Informed choice is essential in healing/recovery from trauma (ATT) & 0 & $24(6)$ & $364(94)$ \\
\hline $\begin{array}{l}\text { Trauma Informed Practice is essential for working with our consumers and } \\
\text { their families (ATT) }\end{array}$ & $10(2.5)$ & $60(15)$ & $318(82)$ \\
\hline I have a comprehensive understanding of Trauma Informed Practice (K) & $172(44)$ & $133(34)$ & $83(22)$ \\
\hline I believe and support the principles of Trauma Informed Practice (K) & $9(2.5)$ & $\begin{array}{l}\text { I don't know what they are } \\
275 \text { (70.5) }\end{array}$ & $104(27)$ \\
\hline $\begin{array}{l}\text { I share my experience and collaborate effectively with colleagues } \\
\text { regarding the use of Trauma Informed Practice (ATT) }\end{array}$ & $6(1.5)$ & $\begin{array}{l}\text { I do not have experience } \\
296 \text { (76) }\end{array}$ & $85(22.5)$ \\
\hline I would like to receive more training on Trauma Informed Practice (ATT) & $7(2)$ & $86(22)$ & $296(76)$ \\
\hline I practice self-care (taking care of my own needs and well-being) (ATT) & $6(1.5)$ & $71(18.5)$ & $312(80)$ \\
\hline $\begin{array}{l}\text { I feel competent to respond calmly and without judgment to a consumer's } \\
\text { strong emotional distress (CO) }\end{array}$ & $5(1.5)$ & $84(21.5)$ & $300(77)$ \\
\hline $\begin{array}{l}\text { I feel competent to engage with traumatized consumers so that they feel } \\
\text { comfortable talking to me (CO) }\end{array}$ & $28(7)$ & 111 (28.5) & 250 (64.5) \\
\hline $\begin{array}{l}\text { I understand how traumatic stress may present itself differently in } \\
\text { consumers of different ages, gender, or cultures. (K) }\end{array}$ & $2(.5)$ & $36(9.5)$ & $351(90)$ \\
\hline $\begin{array}{l}\text { It is reasonable to assume that consumers who are homeless have been } \\
\text { exposed to trauma }(\mathrm{K})\end{array}$ & $60(15.5)$ & $111(28.5)$ & $218(56)$ \\
\hline
\end{tabular}


Table 3. Statistical differences between clinical and non-clinical staff for trauma statements

\begin{tabular}{|c|c|c|c|c|}
\hline Statement & Clinical (\%) & Non-clinical (\%) & $p$ & phi \\
\hline \multirow{3}{*}{ Exposure to trauma is common } & Disagree $=8$ & Disagree $=21$ & \multirow{3}{*}{$>.005$} & \multirow{3}{*}{.29} \\
\hline & Undecided $=10$ & Undecided = 25 & & \\
\hline & Agree $=82$ & Agree $=54$ & & \\
\hline \multirow{3}{*}{$\begin{array}{l}\text { Trauma affects physical, emotional and } \\
\text { mental well-being }\end{array}$} & Disagree $=0$ & Disagree $=1$ & \multirow{3}{*}{$>.005$} & \multirow{3}{*}{.23} \\
\hline & Undecided $=0$ & Undecided $=8$ & & \\
\hline & Agree $=100$ & Agree $=91$ & & \\
\hline \multirow{3}{*}{$\begin{array}{l}\text { There is a connection between mental health } \\
\text { issues and past traumatic experiences or } \\
\text { adverse childhood experiences }\end{array}$} & Disagree $=1$ & Disagree $=0$ & \multirow{3}{*}{$>.005$} & \multirow{3}{*}{.23} \\
\hline & Undecided $=2$ & Undecided = 13 & & \\
\hline & Agree $=97$ & Agree $=87$ & & \\
\hline \multirow{3}{*}{ Re-traumatisation can occur unintentionally } & Disagree $=0$ & Disagree $=0$ & \multirow{3}{*}{$>.005$} & \multirow{3}{*}{.17} \\
\hline & Undecided $=6$ & Undecided = 16 & & \\
\hline & Agree $=94$ & Agree $=84$ & & \\
\hline \multirow{3}{*}{$\begin{array}{l}\text { Informed choice is essential in } \\
\text { healing/recovery from trauma }\end{array}$} & Disagree $=0$ & Disagree $=0$ & \multirow{3}{*}{.01} & \multirow{3}{*}{.13} \\
\hline & Undecided $=4$ & Undecided = 10 & & \\
\hline & Agree $=96$ & Agree $=90$ & & \\
\hline \multirow{3}{*}{$\begin{array}{l}\text { I believe and support the principles of } \\
\text { Trauma Informed Practice }\end{array}$} & Disagree $=2$ & Disagree $=2$ & \multirow{3}{*}{$>.005$} & \multirow{3}{*}{.18} \\
\hline & I don't know what they are $=65$ & I don't know what they are $=82$ & & \\
\hline & Agree $=33$ & Agree $=16$ & & \\
\hline \multirow{3}{*}{$\begin{array}{l}\text { I share my experience and collaborate } \\
\text { effectively with colleagues regarding the use } \\
\text { of Trauma Informed Practice }\end{array}$} & Disagree $=1$ & Disagree $=1$ & \multirow{3}{*}{$>.005$} & \multirow{3}{*}{.16} \\
\hline & I do not have experience $=72$ & I do not have experience $=86$ & & \\
\hline & Agree $=27$ & Agree $=13$ & & \\
\hline \multirow{3}{*}{$\begin{array}{l}\text { I would like to receive more training on } \\
\text { Trauma Informed Practice }\end{array}$} & Disagree $=1$ & Disagree $=5$ & \multirow{3}{*}{$>.005$} & \multirow{6}{*}{.18} \\
\hline & Undecided $=19$ & Undecided $=28$ & & \\
\hline & Agree $=80$ & Agree $=68$ & & \\
\hline \multirow{3}{*}{$\begin{array}{l}\text { I feel competent to engage with traumatised } \\
\text { consumers so that they feel comfortable } \\
\text { talking to me }\end{array}$} & Disagree $=5$ & Disagree $=11$ & \multirow{3}{*}{$>.005$} & \\
\hline & Undecided $=24$ & Undecided $=37$ & & \\
\hline & Agree $=71$ & Agree $=52$ & & \\
\hline
\end{tabular}

Section two of the survey was for clinical staff only ( $\mathrm{n}=$ 254) and sought levels of agreement about six statements regarding trauma-informed practice. Responses are shown in Table 4. There were high levels of neutral responses for statements about effective screening measures and perceived competence to provide trauma focused interventions. All the questions in Table 4 relate to practice.

Table 4. Levels of agreement for statements about trauma-informed practice (clinical staff)

\begin{tabular}{|c|c|c|c|}
\hline Statement & $\begin{array}{l}\text { Disagree } \\
\text { n (\%) }\end{array}$ & $\begin{array}{l}\text { Undecided } \\
\text { n (5) }\end{array}$ & $\begin{array}{l}\text { Agree } \\
\text { n (5) }\end{array}$ \\
\hline My organisation encourages use of effective screening measures for assessing traumatic stress & $60(24)$ & $139(55)$ & $53(21)$ \\
\hline My organisation is flexible in the way they provide care to make it less stressful for consumers. & $21(8)$ & $71(28)$ & $160(64)$ \\
\hline Health care professionals should regularly assess clients for symptoms of traumatic stress. & $2(1)$ & $31(12)$ & $219(87)$ \\
\hline I have colleagues I can turn to for help with a client experiencing significant traumatic stress. & $45(18)$ & $42(17)$ & $165(65)$ \\
\hline I feel competent to provide basic trauma-focused interventions & $46(18)$ & $107(42)$ & $99(40)$ \\
\hline In the last six months I have asked a client questions to assess his/her symptoms of distress & $87(35)$ & $21(8)$ & 144 (57) \\
\hline
\end{tabular}

The final section of the survey was a capacity assessment, measuring clinical staff's perceived level of importance for processes regarding family violence, homelessness and trauma-informed care compared to their own confidence in the same domains. It is a gap analysis of the importance of the domain and staff self-reported confidence in their skill and understanding.

In terms of importance the median score for all responses shows staff rated each item very highly, with all staff rating 'Gauging the level of risk for a person experiencing 
family violence' as important. The lowest level of confi- is in accessing resources for trauma-informed care followed dence in skills or understanding was in 'Accessing resources for trauma-informed care'. The gap scores between the domains are shown in Figure 1 from lowest gap to highest gap. The highest gap between importance and confidence by trauma-informed communication skills. The lowest gap between importance and confidence is in documenting a patient's experience of family violence, followed by identifying risk factors of family violence.

\begin{tabular}{|c|c|c|c|}
\hline & Importance & - Gap & Confidence \\
\hline $\begin{array}{l}\text { Documenting a patient's experience of family violence } \\
\text { (including use of the hospital's systems) }\end{array}$ & 2.93 & 0.6 & 2.33 \\
\hline Identifying risk indicators of family violence & 2.97 & 0.62 & 2.35 \\
\hline Identifying risk factors for homelessness & 2.93 & 0.72 & 2.21 \\
\hline $\begin{array}{l}\text { Providing referrals to appropriate services or supports for } \\
\text { people who report family violence }\end{array}$ & 2.96 & 0.77 & 2.19 \\
\hline $\begin{array}{l}\text { Gauging the level of risk for a person experiencing family } \\
\text { violence }\end{array}$ & 3 & 0.82 & 2.18 \\
\hline $\begin{array}{l}\text { Providing referrals to appropriate services or supports for } \\
\text { people who report they are homeless }\end{array}$ & 2.99 & 0.89 & 2.1 \\
\hline Staff training in trauma-informed communication skills & 2.87 & 1 & 1.87 \\
\hline Accessing resources for trauma-informed care & 2.91 & 1.07 & 1.84 \\
\hline
\end{tabular}

Figure 1. Gap analysis of perceived importance and staff confidence in trauma-informed practice, family violence and responding to homelessness

Staff were invited to write any comments on completion of the survey questions. There were 17 staff who commented. The majority of comments related to the need for education training or resources, and included:

'I have not had sufficient training in trauma informed care' 'I would benefit from more education'

'We need training in all areas of the health service in this topic'

'Resources are vague or non-existent'

'Training needs to be done by local people with local solutions'

\section{Discussion}

This paper presented the results of a descriptive study of rural health workforce knowledge, attitudes and awareness of trauma and the self-reported capacity to respond. The focus was to further understand service engagement to vulnerable groups, particularly those impacted by homelessness and family violence. The high response rate of $78 \%$ indicates that the perceptions of most staff employed by the health services are captured in the survey. The majority of staff who participated in the study identified as nurses. Nurses are the major employee group of health services, so this finding is not unexpected. Only $35 \%$ of participants were from non-clinical backgrounds.
Barriers to health care for those impacted by homelessness and family violence include previous encounters and experience with staff providing care. ${ }^{[18,19]}$ Two Australian studies, ${ }^{[20,21]}$ espouse the need for staff working with homeless people to be trained in trauma-informed care. It is reasonable to assume that people who are homeless have been exposed to a traumatising event, ${ }^{[9]}$ either as the cause for homelessness (such as domestic violence) or homelessness itself being a traumatic event. Many people experiencing homelessness also suffer from depression, substance abuse, and severe mental illness. These issues leave individuals even more vulnerable to re-victimisation, interfere with their ability to work, impair their social networks, and further complicate their service needs. ${ }^{[22]}$

Evidence is emerging that the current pandemic will cause increasing rates of homelessness and exposure to family violence, while restrictions limit access to the usual services that support vulnerable people. ${ }^{[23]}$ The COVID-19 pandemic is in itself a potentially traumatic event. It has caused abrupt changes in life circumstances; uncertainty about the future; deterioration of livelihood; restriction of social contacts; imposed quarantine; stigmatisation, discrimination and fragmentation of communities; loss of loved ones; deprivation of culturally appropriate mourning rituals; and finally, the 
threat of contracting COVID-19. ${ }^{[24]}$ That lack of predictability paired with lack of control is compounded for victims and survivors of trauma whose anxiety responses can be triggered by the overall social climate of uncertainty and worry. ${ }^{[23]}$ Although this study was planned pre-pandemic, the trauma resulting from COVID-19 suggests that trauma-informed principles of care should be prioritised by health services.

Although there were many staff who reported undertaking training in family violence, only a small proportion of respondents reported having undertaken training in traumainformed care and $44 \%$ disagreed that they had an understanding of trauma-informed practice. The majority of respondents also reported that they did not know the principles of, or had experience in, trauma-informed practice and $76 \%$ reported wanting to receive more training in trauma-informed practice. This identifies a clear desire for staff training. The extant literature suggests that there is a continuum from being trauma aware (seeking information out about trauma and its implications for organisations) to being trauma-informed (a cultural shift at the systemic level). ${ }^{[8]}$

There were high rates of awareness of the effects of trauma, with the majority agreeing with knowledge and awareness statements. Multiple studies have reported negative effects associated with experiencing trauma across all facets of life. Mental ill-health, physical illness, social and relational difficulties, have all been linked to previous traumatic experiences. $^{[25]}$ As expected, non-clinical staff members had lower levels of knowledge and awareness of trauma, possibly due to less experience with people experiencing trauma.

When respondents were asked about supporting the principles of trauma-informed practice and sharing experience with colleagues regarding the use of trauma-informed practice, more than $70 \%$ reported that they did not know the principles, and that they did not have experience. These responses correlate with the comments provided by staff that more training and education is desired.

Section two of the survey focused on clinical staff and presented statements about practice, confidence and competency. The majority of staff (55\%) reported that they were undecided about whether the organisation encouraged effective screening measures for assessing traumatic stress, and only $21 \%$ agreed with the statement. At the same time, $87 \%$ agreed that health professionals should undertake assessments for traumatic stress. This finding suggests that clinical staff, predominantly nurses, could improve practice by implementing screening measures in order to respond appropriately to people experiencing the effects of trauma.

Training organisations in trauma care emphasises the importance of maintaining constant attention and awareness Published by Sciedu Press toward the potential impacts of traumatic experiences across all practices. ${ }^{[1]}$ Studies that report the impact of staff training in trauma-informed care, including the use of screening tools, demonstrate impressive patient outcomes such as decreased use of restraints, reduction of property destruction, and improved patient satisfaction. ${ }^{[26]}$

The capacity assessment (or gap analysis) supports the previous findings in this study of the need for more education and training, with the largest gaps found in trauma-informed communication skills and accessing resources for traumainformed care. All of the domains in the capacity assessment were perceived as important by greater than $60 \%$ of respondents (ranged from 60\%-67\%). However, when reporting their confidence for having sufficient skills and understanding of the same domains less than $35 \%$ reported confidence (ranged from $11 \%-34 \%$ ). Although there are gaps in all domains, generally a gap analysis identifies and addresses the most critical gaps as a priority - in this instance resources and training, followed by referral pathways for family violence, gauging risk in family violence, referral pathways for homeless people, identifying risk factors for homelessness, risk indicators of family violence and finally documentation of family violence. As more evidence surfaces regarding the significance of trauma on physical and psychological well-being the idea of practicing through a trauma-informed lens has become particularly important. ${ }^{[27]}$

The ubiquitous exposure to COVID-19 argues for all health systems to use a trauma-informed response as a universal precaution. ${ }^{[28]}$ That begins with incorporating training in trauma-informed principles, the development of resources and clear pathways to care in its response.

\section{Conclusion}

This survey provided a basis of staff knowledge, awareness, confidence and capacity to respond to consumers with a history of trauma. The survey identified that staff were trauma aware but not confident in trauma-informed practice. The study showed a clear need, and desire, for training in traumainformed care and specifically a need for resources and clear referral pathways to respond to trauma. There is also a need for implementation of broad organisational policies to support a culture of providing trauma-informed care.

\section{ACKNOWLEDgements}

The principal researcher is supported through the University Department of Rural Health Multi-disciplinary Training Program. The research was conducted on lands traditionally owned by the Yorta Yorta Nations.

\section{Conflicts of InTEREST Disclosure}

The authors declare that there is no conflict of interest. 


\section{REFERENCES}

[1] Department of Health, Substance Abuse and Mental Health Services Administration and USA, "Concept of Trauma and Guidance for a Trauma-Informed Approach. SAMHSA. 2014. [Online]. Available from: https://ncsacw.samhsa.gov/userfiles/files/SAMH SA_Trauma.pdf

[2] Anda RF, et al. The enduring effects of abuse and related adverse experiences in childhood. Eur. Arch. Psychiatry Clin. Neurosci. 2006; 256(3): 174-186. PMid:16311898 https://doi.org/10.1007/ s00406-005-0624-4

[3] Kezelman C, Hossack N, Stavropoulos P, et al. The cost of unresolved childhood trauma and abuse in adults in Australia. 2015. Available from: https://www.pc.gov.au/__data/assets/pdf_file/0 019/239014/sub047-mental-health.pdf

[4] Berger E, Martin K. Why every teacher needs to know about childhood trauma. The Conversation. 2020. Available from: https : //theconversation.com/why-every-teacher-needs-to-k now-about-childhood-trauma-132965\#: : text=Bydevel opingknowledgeaboutthe, andreducedriskof burnout

[5] Mills KL, et al. Assessing the Prevalence of Trauma Exposure in Epidemiological Surveys. Aust. New Zeal. J. Psychiatry. 2011; 45(5): 407-415. PMid:21189046 https://doi .org/10.3109/00 048674.2010 .543654

[6] Rosenman S. Trauma and Posttraumatic Stress Disorder in Australia: Findings in the Population Sample of the Australian National Survey of Mental Health and Wellbeing. Aust. New Zeal. J. Psychiatry. 2002; 36(4): 515-520. PMid:12169152 https ://doi.org/10.1046/j . 1440-1614.2002.01039.x

[7] Australian Institute of Health and Welfare. Stress and Trauma: Snapshot. Determinants of Health. 2020. [Online]. Available from: https://www.aihw.gov.au/reports/australias-hea 1th/stress-and-trauma

[8] Wall L, Higgins D, Hunter C. Trauma-informed care in child/family welfare services. Child Fam. Community Aust. 2016; 37: 1-19.

[9] Hopper EK, Bassuk EL, Olivet J. Shelter from the Storm: TraumaInformed Care in Homelessness Services Settings. Open Health Serv. Policy J. 2010; 3(2): 80-100. https ://doi .org/10.2174/1874 924001003020080

[10] Markoff LS, Reed BG, Fallot RD, et al. Implementing TraumaInformed Alcohol and Other Drug and Mental Health Services for Women: Lessons Learned in a Multisite Demonstration Project. Am. J. Orthopsychiatry. 2005; 75(4): 525-539. PMid:16262512 https://doi.org/10.1037/0002-9432.75.4.525

[11] Melz H, Morrison C, Ingoldsby E, et al. Review of trauma-informed initiatives at the system level. Trauma-informed approaches: Connecting research, policy and practice to build resilience in children and families. James Bell Associates, Arlington USA for Office of the Assistant Secretary for Planning and Evaluation U.S. Department of Health and Human Services. 2019. [Online]. Available from: https://aspe.hhs.gov/system/files/pdf/262051/T I_Approaches_Research_Review.pdf

[12] Stokes Y, Jacob JD, Gifford W, et al. Exploring Nurses' Knowledge and Experiences Related to Trauma-Informed Care. Glob. Qual. Nurs Res. 2017.

[13] Fleishman J, Kamsky H, Sundborg S. Trauma-Informed Nursing Practice. Online J. Issues Nurs. 2019; 24(2).
[14] King S, Chen KLD, Chokshi B. Becoming Trauma Informed: Validating a Tool to Assess Health Professional's Knowledge, Attitude, and Practice. Pediatr. Qual. Saf., 2019; 4(5). PMid:31745518 https://doi.org/10.1097/pq9.0000000000000215

[15] Bruce MM, Kassam-Adams N, Rogers M, et al. Trauma Providers' Knowledge, Views, and Practice of Trauma-Informed Care. J. Trauma Nurs. 2018; 25(2): 131-138. PMid:29521782 https ://doi.org/ $10.1097 /$ JTN .0000000000000356

[16] IBM Corp. IBM SPSS Statistics for Windows. IBM, Armonk, New York; 2016.

[17] Cohen J. Statistical Power Analysis for the Behavioral Sciences. New York, NY: Routledge Academic; 1988.

[18] Paisi M, et al. Barriers and enablers to accessing dental services for people experiencing homelessness: A systematic review. Community Dent. Oral Epidemiol. 2019; 47(2). PMid:30614026 https : //doi.org/10.1111/cdoe.12444

[19] Brown A, Rice S, Rickwood D, et al. Systematic review of barriers and facilitators to accessing and engaging with mental health care among at-risk young people. Asia-Pacific Psychiatry. 2015.

[20] Wood L, Wood N, Vallesi S, et al. Hospital collaboration with a Housing First program to improve health outcomes for people experiencing homelessness. Housing, Care Support. 2019; 22(1): 27-39. https ://doi.org/10.1108/HCS-09-2018-0023

[21] Davies A, Wood L. Homeless health care: meeting the challenges of providing primary care. Med. J. 2018.

[22] Jainchill N, Hawke J, Yagelka J. Gender, psychopathology, and patterns of homelessness among clients in shelter-based TCs. Am J Drug Alcohol Abus. 2000; 26: 553-67. PMid:11097192 https: //doi.org/10.1081/ADA-100101895

[23] Collin-Vézina D, Brend D, Beeman I. When it counts the most: Trauma-informed care and the COVID-19 global pandemic. Dev. Child Welf. 2020. https://doi.org/10.1177/251610322094 2530

[24] Javakhishvili JD, Ardino V, Bragesjö M, et al. Trauma-informed responses in addressing public mental health consequences of the COVID-19 pandemic: position paper of the European Society for Traumatic Stress Studies (ESTSS). Eur. J. Psychotraumatol. 2020; 11(1): 1780782. PMid:33029320 https : //doi.org/10.1080/20 008198.2020 .1780782

[25] Nanda R, et al. The enduring effects of abuse and related adverse experiences in childhood: A convergence of evidence from neurobiology and epidemiology. Eur. Arch. Psychiatry Clin. Neurosci. 2006; 256: 174-186. PMid:16311898 https://doi.org/10.1007/s0 0406-005-0624-4

[26] German M, Crawford D, Dumpert K. Measuring the impact of trauma informed primary care: are we missing the forest for the trees? Cent. Heal. care Strateg. 2020.

[27] Abdoh N, Bernardi E, Mccarthy A. Knowledge, Attitudes, and Practices of Trauma Informed Practice: A survey of health care professionals and support staff at Alexander Street Community. NURS Synth. Proj. 2017.

[28] Griffin G. Defining trauma and a trauma-informed COVID-19 response. Psychol. Trauma Theory, Res. Pract. Policy. 2020; 12(S1): S279-S280. PMid:32551754 https://doi.org/10.1037/tra0 000828 